\title{
Oral Helicobacter pylori: Interactions with host and microbial flora of the oral cavity
}

\section{Helicobacter pylori w jamie ustnej - interakcje z mikrobiotą gospodarza}

\author{
Paweł Krzyżek, ${ }^{1, A-D, F}$, Grażyna Gościniak, ${ }^{1, E}$ \\ Department of Microbiology, Wroclaw Medical University, Poland \\ A - research concept and design; $\mathrm{B}$ - collection and/or assembly of data; $\mathrm{C}$ - data analysis and interpretation; \\ $D$ - writing the article; $E$ - critical revision of the article; $F$ - final approval of the article
}

\section{Address for correspondence \\ Paweł Krzyżek}

E-mail: krojcerpawel@gmail.com

\section{Funding sources}

None declared

Conflict of interest

None declared

Received on September 18, 2017

Revised on November 21, 2017

Accepted on December 16, 2017

\begin{abstract}
The role of the oral cavity as a reservoir for Helicobacter pylori is still a controversial issue. There is a great number of articles indicating the presence of this pathogen in the oral cavity, but discrepancies among techniques for $\mathrm{H}$. pylori detection and the variations in the patients tested often make it difficult to formulate a final verdict. Outer membrane proteins (OMPs) are one of the most important factors determining colonization of H. pylori in the oral cavity. Among them, the key role is attributed to BabA, SabA and NapA, all of which promote adherence and retention within this area. The oral cavity is characterized by the co-existence of numerous microorganisms which may potentially affect the physiology and morphology of $\mathrm{H}$. pylori. The presence of coccoid-stimulating factors and relatively low levels of Al-2 in the early- to mid-stages of supragingival plaque allow dental H. pylori to colonize this niche as nonculturable spherical forms. On the other hand, subgingival plaque characterized by high numbers of periopathogens, capable of synthesizing high concentrations of Al-2, may favor the presence of mixed populations of spiral and coccoid $H$. pylori forms. This review article provides an up-to-date knowledge about the ability of oral H. pylori to interact both with the host and the local microflora of the oral cavity.
\end{abstract}

Key words: biofilm, Helicobacter pylori, oral cavity, dental plaque, microflora

Słowa kluczowe: biofilm, Helicobacter pylori, jama ustna, płytka nazębna, mikroflora

DOI

$10.17219 / \mathrm{dmp} / 81259$

Copyright

() 2018 by Wroclaw Medical University

and Polish Dental Society

This is an article distributed under the terms of the

Creative Commons Attribution Non-Commercial License

(http://creativecommons.org/licenses/by-nc-nd/4.0/) 
Every living organism, to maintain the stability of its internal environment, must constantly adapt at the molecular, cellular and physiological levels. The gastrointestinal tract is the largest surface of the body exposed to the external environment. Therefore, the presence of mucous membranes and a well-functioning immune system is essential to protect the host organism. ${ }^{1}$

The first barrier in the digestive system, including the oral cavity, is the mucus layer. The mucus matrix forms a strongly hydrophilic colloid, mainly composed of water (about 95\%), but also lipids (phospholipids, cholesterol), nucleic acids, polysaccharides and proteins (antibodies, defensins, growth factors). ${ }^{2}$ Antibodies found in saliva belong mainly to 2 classes, i.e., IgA (90-98\%) and IgG (1-10\%). Their presence contributes to the inactivation and opsonization of microorganisms, which are then detected by the antigen presenting cells (APCs) and phagocyted. In the oral cavity, antibody production comes through the stimulation of B cells, located within the mucosa-associated lymphoid tissue (MALT). The main region of their recruitment is nasopharynxassociated lymphoid tissue (NALT), which consists of the adenoids and palatine tonsils of Waldeyer's ring. Salivary defense proteins play an important role in the protection of the oral environment. These include, but are not limited to: lysozyme, bactericidal increasing proteins (BPIs), palate lung and nasal epithelial clone proteins (PLUNCs), salivary amylase, cystatins, proline-rich proteins, statherin and peroxidases. The function of these proteins is to adsorb to the microbial surface and form ion channels, which leads to the microorganism's destruction. In addition, some of these compounds also possess immunomodulating activity and the ability of chelating metal ions from the local environment $\left(\mathrm{Fe}^{3+}, \mathrm{Ni}^{2+}, \mathrm{Cu}^{2+}\right) .^{3}$

Another important component of mucus is the high molecular weight oligomeric glycoproteins, namely mucins. More than 20 mucins covering the epithelial surface of the respiratory, digestive and reproductive tracts have been detected in the human body. ${ }^{4} \mathrm{MUC} 1$, MUC4, MUC19, MUC5B and MUC7 are produced in the oral cavity, while the dominant and most important groups are the last two. ${ }^{4,5}$ MUC5B is a compound with a molecular weight in excess of $1000 \mathrm{kDa}$ and consists of disulfide-bonded subunits. ${ }^{3}$ MUC5B is a member of the gel-forming mucin family, secreted by the submandibular, sublingual, palatine and labial mucous cells of the salivary glands. ${ }^{4}$ MUC7 is a secretory mucin present in the form of monomers (180-200 kDa) or dimers. It is located mainly in the submandibular and sublingual salivary glands. ${ }^{3,4}$ Mucins and defense proteins play a crucial role in the formation of acquired pellicle, i.e., a thin layer $(0.5-1 \mu \mathrm{m})$ of saliva components with the ability to bind to calcium hydroxide. These components have receptors for microbes and, thus, in the surface immune exclusion process, con- tribute to the immobilization of microorganisms and protection against host tissue invasion. ${ }^{3,5}$ Despite the protective function, this process also has a disadvantageous aspect associated with the formation of biofilm by microorganisms colonizing the oral cavity. ${ }^{3,4}$

Populations of microbes often form highly organized complexes called biofilms. These structures are made up of microbial cells embedded within the extracellular matrix, which accounts for nearly $90 \%$ of biofilm and consists of exopolysaccharides, proteins, lipids and extracellular DNA (eDNA). Biofilm provides a protective environment against adverse conditions including temperature, $\mathrm{pH}$ and oxidation-reducing potential fluctuations, presence of antimicrobial agents (antibiotics, enzymes) and the action of innate and adaptive immune systems. Moreover, besides the protective function, biofilm also determines co-adhesion and aggregation of the cells, formation of a strongly hydrated environment (protection against drying) and sorption of organic and inorganic compounds from the surrounding environment. ${ }^{6}$ The formation of biofilm is also related to another important process, supervising the physiology of microorganisms, called quorum sensing (QS). In the course of this mechanism, microbes communicate using small molecular signaling molecules, which allow them to sense their density and integrate actions that resemble multicellular organisms. ${ }^{7}$ With respect to the intestinal flora, the oral cavity is one of the most colonized areas in the human body. The number of bacterial species localized in this region is estimated at $600-800 .{ }^{8}$ In addition to the enormous diversity of microorganisms, the oral ecosystem is characterized by succession, microbial composition variations and changes in conditions within the different morphological regions of the oral cavity. ${ }^{9}$ For this reason, oral biofilm is a very dynamic environment modified by availability and type of nutrient substances, $\mathrm{pH}$, microbial toxic metabolite concentration, host immune response and shear forces. ${ }^{10}$ The surface of the teeth is covered by the saliva components forming the acquired pellicle. ${ }^{9}$ This area is colonized by microorganisms capable of linking to these components, referred to as early colonizers, i.e., Streptococcus spp., Actinomyces spp., Gemella spp., Veillonella spp., Selenomonas spp., and Capnocytophaga spp. ${ }^{8-11}$ Fusobacterium nucleatum is known to act as the "bridge bacterium". This is due to the long, filamentous shape and numerous multivalent adhesins exposed on the surface of this bacterium. These features make it possible to co-adhere with Streptococcus spp. and recruit Gram-negative rods (late colonizers), including: Porphyromonas gingivalis, Tannerella forsythia, Treponema denticola, and Aggregatibacter actinomycetemcomitans. ${ }^{8,12}$ In many scientific studies, it is also suggested that the oral cavity may be a reservoir site for Gram-negative, microaerophilic Helicobacter pylori. ${ }^{5,13-17}$ 


\section{Colonization of the oral cavity by H. pylori}

H. pylori infections occur most often in childhood, and clinical symptoms may develop over many years. This microorganism inhabits the gastric mucosa, where it is able to survive despite the therapy used. Persistent infections induce chronic gastritis, which is associated with the risk of developing gastric or duodenal ulcers and gastric cancers. ${ }^{18}$ Transmission from person to person seems to be the main mechanism of the bacteria spreading. It is suggested that environmental reservoirs, such as contaminated water and food or infected animals, also exist. The factors influencing the frequency of $H$. pylori infection include age, sex, genetic predisposition, immunological status, and hygiene level. ${ }^{16}$

The outer membrane proteins (OMPs) presence is a crucial factor determining host colonization by $H$. pylori. It is estimated that $4 \%$ of the genome of this bacterium encodes information for the production of these structures. ${ }^{19}$ The ability of direct adhesion to host epithelial cells is a process responsible for persistent infections, because it contributes to the protection against the removal of microbes from the mucous layer and enables delivery of virulence factors to eukaryotic cells. ${ }^{19,20}$

One of the most important $H$. pylori adhesins is BabA (blood group antigen binding adhesin A), which allows interaction with fucosylated Lewis $\mathrm{b}\left(\mathrm{Le}^{\mathrm{b}}\right)$ and $\mathrm{H}$ type 1 antigens. For this reason, the protein has a strong affinity to MUC5AC, mucin secreted in the stomach environment. Additionally, BabA also determines adhesion with saliva components, i.e., MUC5B, proline-rich proteins and salivary agglutinin (gp-340). ${ }^{20}$

The second essential adhesin produced by $H$. pylori is SabA (sialic acid-binding adhesin). This protein promotes the adherence of bacteria to the inflamed stomach tissue, which is characterized by increased mucin sialylation (the process of linking sialic acid residues to Lewis antigens). This property also affects the ability of $H$. pylori to associate with salivary glycoproteins, such as MUC5B, MUC7, carbonic anhydrase VI, zinc- $\alpha$-2-glycoprotein or heavy chains of $\operatorname{sigA1}$. The expression of SabA is regulated by the $\mathrm{pH}$ of the environment, and the amount of $s a b A$ mRNA decreases under acidic conditions. ${ }^{20}$ This feature makes it possible that in more neutral $\mathrm{pH}$ conditions (e.g. in the oral cavity) SabA production can play a key role in H. pylori oral mucosa colonization.

The third vital adhesin expressed by almost all clinical H. pylori strains is NapA (neutrophil-activating protein). This protein has the ability to stimulate myeloperoxidase secretion and the production of reactive oxygen species (ROS) by neutrophils. Therefore, this factor most likely contributes to the release of nutrients from destroyed tissues. ${ }^{21} H$. pylori NapA has been shown to be capable of binding to the sulfur oligosaccharides of saliva, i.e., sulfo-Le ${ }^{\mathrm{a}}$, sulfo-galactose and sulfo- $N$-acetyl-glucosamine. ${ }^{22}$
The significance of these proteins was confirmed by an oral $H$. pylori virulence factors analysis, because the presence of all these adhesins was detected in this microbe. Furthermore, the ability of oral $H$. pylori strains to secrete factors associated with tissue damage, inflammation initiation and nutrient uptake, i.e., VacA (vacuolating cytotoxin A), CagA (cytotoxin-associated antigen A), and serine protease HtrA, was observed. ${ }^{23}$ The ability of $H$. pylori to cause pathological changes in the oral cavity is uncertain. In the stomach environment, this bacterium has many factors facilitating persistent colonization of the gastric mucosa. These properties include the ability to invade eukaryotic cells, disrupt cell-cell contact, promote inflammation, inhibit immune cell activity (including $\mathrm{T}$ cells) and induce excessive host cell proliferation. ${ }^{24} \mathrm{H}$. pylori colonizing the oral cavity is also suggested to be involved in the development of various ailments in this region, such as halitosis, glossitis, recurrent aphthous stomatitis and dental caries. ${ }^{25}$ Studies on the human acute monocytic leukemia cell line (THP-1) have shown that these microorganisms may be responsible for causing pathological changes in the oral cavity. The main factor responsible for this effect was CagA, because strains producing this protein contributed to significant increases in inflammatory cytokine secretion (IL-6, IL-8, IFN- $\gamma$ ) as compared to non-producing strains. ${ }^{26}$ The capacity of $H$. pylori to interact with the host and modulate the local environment was also demonstrated by the ability of this bacterium to induce elevated MUC5B and MUC7 levels by three- and twofold, respectively. The increase in the amount of these components, which are receptors for oral H. pylori, can promote colonization and retention within the oral cavity. ${ }^{27}$ Despite this, there is a need to increase the number of studies showing a direct relationship between $H$. pylori infection/colonization and oral pathophysiology.

\section{Epidemiology of oral H. pylori infections}

There are many diagnostic methods for the detection of $H$. pylori, but none of them are $100 \%$ specific and sensitive. Invasive methods require removal of the gastric mucosa. Histopathological examination allows for accurate assessment of the degree and type of the gastric mucosa inflammation. Hematoxylin and eosin staining are used for the visualization of inflammatory cells, while Giemsa or Genta staining serve for microorganism detection. The sensitivity of the method is $89-91 \%$ and the specificity is $91-100 \%$. Culture methods have a very high specificity (about 100\%), but much lower sensitivity (78-94\%). In addition, the culture of this bacterium is necessary to determine the sensitivity of $H$. pylori to antibiotics. Rapid urease testing is the simplest, cheapest and fastest test determining urease activity in biopsy specimens. This method has a sensitivity of $82-94 \%$ and 
a specificity of $96-96 \%$. PCR is a highly useful method because, apart from the detection of bacteria (the highest accuracy in $16 \mathrm{~S}$ rRNA gene detection), it also provides information about the production of virulence markers, such as cagA or vacA. Improved PCR methods with colorimetric detection of $H$. pylori DNA using isothermal helicase-dependent amplification result in sensitivity and specificity of up to $95 \%$. Non-invasive methods are divided into active (detecting active $\mathrm{H}$. pylori infection) or passive (showing evidence of contact with bacteria). Among the active assays are: the fecal antigen test which is used to determine the presence of H. pylori antigens in feces (sensitivity and specificity $92 \%$ and $94 \%$, respectively), and the urease test. The latter is based on the detection of $H$. pylori urease activity during active infection. The level of labeled $\mathrm{CO}_{2}$ derived from the decomposition of the labeled urea ${ }^{13} \mathrm{C}$ is measured. Sensitivity and specificity are $91 \%$ and $94 \%$, respectively. Serological tests belong to passive assays and are based on the detection of IgG anti-H. pylori antibody levels in the plasma. The biggest disadvantage of these tests is the lack of the ability to delineate between active infection and temporal exposure to H. pylori antigens. This method is characterized by a high sensitivity (90-97\%), but a low specificity $(50-96 \%) .{ }^{28,29}$

The role of the oral cavity as a reservoir for H. pylori is still a controversial issue. There are a great number of articles indicating the presence of this pathogen in the oral cavity, but discrepancies among techniques for $H$. $p y$ lori detection and the variations in patients tested often make it difficult to formulate a final verdict. One of the newest and more accurate meta-analyzes conducted by Sayed et al. suggests that dental plaque can be an alternative, extragastric site of $H$. pylori residence. ${ }^{14}$ Briefly, the frequency of $H$. pylori detection depends on the diagnostic technique used. The prevalence of bacteria detection using urease tests in most studies ranged between 50\% and $100 \%$. PCR studies have even greater divergence in results, i.e., $0-100 \%$ (of which only $7 / 35$ studies showed a frequency greater than 50\%). Culture methods have a low sensitivity, with prevalence lower than 50\% (half of studies with prevalence lower than 10\%). Inhomogeneous results were obtained using immunoassays (2 studies with frequencies above $65 \%$ and 2 with very low prevalence, $0 \%$ and $11 \%) .{ }^{14}$

Namiot et al., in oral H. pylori detection, used immunoassays intended routinely for fecal antigen detection. ${ }^{30-32}$ The authors suggest that these tests can be successfully used to detect $H$. pylori in the oral cavity after appropriate sample preparation (preincubation in a microaerophilic atmosphere). This procedure increases the incidence of $H$. pylori more than twice. ${ }^{31}$ Using this method, a high prevalence of these microorganisms in dental plaque was reported, i.e., $58.7 \%{ }^{32}$ and $65.6 \% .^{30}$ It was suggested that plaque reduction and oral health had no effect on the amount of antigens of these bacteria. ${ }^{32}$
Interesting results were obtained using molecular techniques. The presence of $H$. pylori in the oral cavity of patients with gastric $H$. pylori was reported in $46 \%$ of the subjects. Moreover, a relationship between these bacteria and increased approximal plaque index (API) and bleeding on probing (BOP) values has been noted. Within strains colonizing the oral cavity, only $16 \%$ expressed cagA, suggesting that this virulence factor is not critical in causing pathological changes to the oral cavity mucosa. ${ }^{33}$ In another study using PCR, the DNA of oral H. pylori was isolated more frequently in patients with oral ailments (leukoplakia and oral lichen planus; $20 \%$ and $23 \%$, respectively) than in those without these diseases ( $0 \%)$. The potential of these microorganisms to promote inflammatory conditions in the oral environment was proposed on this basis. ${ }^{34}$

There is also a relationship between the presence of pathological gingival pockets and $H$. pylori infections. Umeda et al. found that in patients with gastric $H$. pylori infection, the detection frequency of these bacteria in the oral cavity was 4-fold higher (41.2\%) when patients had gingiva with a pocket depth of $\geq 4 \mathrm{~mm}$ than those with healthy periodontium (9.1\%). ${ }^{35}$ Similar observations were made in a large-scale epidemiological study of 4504 patients by Dye et al..$^{36}$ It has been shown that pocket depth greater than $5 \mathrm{~mm}$ correlates positively with $H$. pylori seroprevalence. Despite the ability of $H$. pylori to colonize dental plaque, this area is not the only site of colonization. Other regions include the tonsils and dorsal mucosa of the tongue. . $^{15,37}$

Many studies based on genetic or serological tests in H. pylori detection have shown the presence of this bacterium in the oral cavity or stomach, while the number of studies demonstrating the effective isolation of this microbe from the oral environment is still very low. Hirsch et al. ${ }^{23}$ successfully managed to isolate live $H$. pylori from samples taken from dental canals. In the morphological observation, using field-emission scanning electron microscopy, the presence of a mixed population of spiral rods and aggregates of coccoids was observed. Cultivation of the samples taken from supragingival plaque was unsuccessful, suggesting that in this niche, H. pylori occurred only in the coccoid form. The presence of particular H. pylori morphological forms in various areas of the oral cavity may indicate that the physicochemical parameters and local microbiological flora may affect the transformation process of $H$. pylori.

\section{Interactions of oral $H$. pylori with microbial flora}

The diversity of environmental factors in the oral cavity contributes to the development of specific microbiota in different regions. ${ }^{11}$ Tooth-associated biofilm can be divided into the supragingival (on the exposed surface of the 
enamel) and subgingival (below the gums and inside the periodontal pocket) regions. ${ }^{9}$

Supragingival plaque is characterized by a low level of species diversity and the dominance of Streptococcus spp., Actinomyces spp. and Lactobacillus spp. ${ }^{38}$ The presence of these microorganisms is related to the intense saccharolytic metabolism associated with the fermentation of carbohydrates to organic acids, and the subsequent acidification of the local environment. In addition to the acidification, the process of supragingival plaque maturation is also linked with the formation of microaerophilic conditions. This is the result of the oxygen consumption by the NADH oxidase in the Embden-Meyerhof-Parnas pathway. This process is accompanied by the appearance of F. nucleatum and late colonizers, which are sensitive to high oxygen levels. ${ }^{11}$

In vitro studies have shown that $F$. nucleatum supports the growth of $P$. gingivalis in the presence of elevated oxygen concentrations (10\% and $20 \%)$ and lack of $\mathrm{CO}_{2}$. Under such conditions, the oxygen-sensitive capnophilic $P$. gingivalis was unable to survive in monoculture. During the co-culture with F. nucleatum, a strong growth of this bacterium was observed. ${ }^{39}$ The ability to generate a capnophilic environment by $F$. nucleatum is a feature that could potentially promote the survival of another bacterium, for which the main factor limiting growth is access to $\mathrm{CO}_{2}$, i.e., H. pylori. ${ }^{40}$ It has been observed that $H$. pylori has a strong capacity to co-aggregate with Fusobacterium spp. naturally isolated from dental plaques (F. nucleatum and F. periodontium). ${ }^{41}$ The tendency to co-aggregate with F. nucleatum was also confirmed in another experiment. ${ }^{42}$ In addition, it has been determined that $P$. gingivalis may also be involved in such interaction and that this is a fimbriae-dependent process. ${ }^{42}$ On this basis, it can be concluded that the presence of $H$. pylori is strongly associated with the physiological functioning of these two bacterial species ( $F$. nucleatum and $P$. gingivalis) within the dental plaque, and vice versa.

Streptococcus spp. is the dominant genus in supragingival plaque. The ability of these bacteria to ferment carbohydrates into acidic products (acetic, formic and lactic acid) reduces local $\mathrm{pH}$ values near 5, during high availability of fermentation substrates. ${ }^{9}$ These are the conditions in which $P$. gingivalis and $F$. nucleatum are unable to survive, because the minimum $\mathrm{pH}$ tolerated by these microorganisms is 6.5 and 5.5 , respectively. ${ }^{11}$ Hence the close association of microbes capable of buffering acidic $\mathrm{pH}$ is highly desirable. The presence of bacteria able to utilize lactic acid, the main fermentation product of Streptococcus spp., may protect the supragingival plaque from excessive acidification. ${ }^{8} H$. pylori has been shown to have genes responsible for the uptake and metabolic conversion of D- and L-lactose. ${ }^{43}$ The $\mathrm{pH}$-buffering process in supragingival plaque can also be mediated in an ammonia-dependent manner. $H$. pylori produces urease, an enzyme which converts urea into $\mathrm{CO}_{2}$ and ammonia.
The urease produced by $H$. pylori has a very high activity. It has been shown that the rate of urea hydrolysis by the enzyme of this bacterium is $36 \pm 28 \mu \mathrm{M} / \mathrm{min} / \mathrm{mg}$ of bacterial proteins. This result is twice as high as that of Proteus mirabilis and 10 times higher than in other urinary tract pathogens. ${ }^{44}$ In vitro studies have demonstrated that the ability to produce ammonia is a key contributor to the preservation of the species diversity in oral biofilm. The presence of physiological concentrations of urea in the culture medium (1-10 $\mathrm{mM}$ urea in saliva) determined the bacterial population heterogeneity. However, in a culture with strains defective in urease production or the absence of urea in the culture medium, there was a drastic decrease in the viability of periopathogens ( $P$. gingivalis, F. nucleatum, Prevotella intermedia), Neisseria subflava and Streptococcus oralis, and the dominance of biofilm by cariogenic bacteria, i.e., Streptococcus spp. (other than S. oralis) and Lactobacillus spp. ${ }^{45}$

Supragingival plaque consists mainly of early colonizers, Streptococcus spp. and Actinomyces spp., which may potentially modulate the physiology of oral $H$. $p y$ lori. It has been observed that such microorganisms have the ability to inhibit $H$. pylori growth in vitro. ${ }^{42}$ Diffusible compounds secreted by Streptococcus mutans ${ }^{46}$ and Streptococcus mitis ${ }^{47}$ contribute to a dramatic decrease in $H$. pylori viability. However, microscopic observation has shown that this effect was caused by the conversion of $H$. pylori to nonculturable coccoid forms. This mechanism is consistent with the lack of possibility to isolate this bacterium from supragingival plaque, in which Streptococcus spp. is the dominant microbial genus. ${ }^{9,23}$ Streptococci are a source of SDSF (Streptococcus diffusible signal factors), which may be involved in the morphological transformation of $H$. pylori into coccoid forms. ${ }^{48}$ In an experiment, Khosravi et al. determined the effect of $H$. pylori and S. mitis co-culture on the gene expression of both bacteria. ${ }^{47}$ It was observed that streptococci expressed genes encoding phosphoglycerate kinase (PGK) only when co-existing with $H$. pylori. PGK is a glycolytic enzyme responsible for the conversion of 3-phosphoglycerol aldehyde to 1,3-bisphosphoglycerate. PGK is a surface protein produced by streptococci in increased amounts during the biofilm phase and is responsible for cell-cell interactions. ${ }^{49}$ During co-culture in $H$. pylori, significant reduction in oxidative stress proteins, including glutathione metabolism enzymes, thioredoxin, flavodoxin and thiol peroxidases, have been observed. On the other hand, the amount of proteins involved in RNA degradation and nucleotide excision repair were increased. ${ }^{47}$ The reduction of genes encoding $H$. pylori antioxidants may potentially be due to increased physical contact between the bacterial cells and PGK-dependent aggregation/biofilm promotion in H. pylori ${ }^{49}$ Despite the use of filters (pore size $0.22 \mu \mathrm{m}$ ) for the spatial separation of both microorganisms, it cannot be excluded that interactions between PGK, present on the surface of membrane vesicles secreted by Strepto- 
coccus $(<0.2 \mu \mathrm{m})$, and $H$. pylori cells still may exist. ${ }^{50}$ The increase in the amount of $H$. pylori proteins responsible for the DNA rearrangement can be beneficial under stressful conditions by rapidly selecting well-adapted strains, for example to antibiotics presence. ${ }^{51,52}$ Because of this, the co-aggregation of both bacteria in dental plaque can promote the survival of $H$. pylori in the oral cavity.

One of the key signaling substances produced within dental plaque is autoinducer- 2 (AI-2). ${ }^{8}$ This compound is a chemorepellent agent that promotes the dispersion of $H$. pylori aggregates/biofilms and initiates negative chemotaxis against the source of these signals. ${ }^{53}$ Hence, the colonization of this niche by $H$. pylori should be impeded or even impossible. Commensal plaque bacteria secrete relatively low concentrations of AI- 2 and respond to much lower levels of these substances in the environment (less than 100 pM) than periopathogens, considered to be strong AI-2 producers (nanomolar concentrations). ${ }^{9}$ The amount of AI-2 produced endogenously by $H$. pylori corresponds to the concentrations of signaling compounds secreted by periopathogenic microbes $(\sim 37 \mathrm{nM}) .{ }^{53}$ Therefore, the presence of coccoid-stimulating factors and relatively low levels of AI-2, in the early- to mid-stages of supragingival plaque allow dental $H$. pylori to colonize this niche as nonculturable spherical forms. On the other hand, subgingival plaque characterized by a high density of periopathogens, and thus also higher concentrations of AI-2, may favor the presence of mixed populations of spiral and coccoid $H$. pylori forms.

Subgingival biofilm is composed of many species of microbes, including the dominance of Actinomyces spp., Tannerella forsythia, Fusobacterium nucleatum, Spirochetes and Synergistetes. ${ }^{38}$ In addition, the presence of $A$. actinomycetemcomitans, Streptococcus parasanguinis, Filifactor alocis, Eubacterium spp., Prevotella spp., Porphyromonas spp., and Campylobacter spp. is also detected. ${ }^{11,54}$ Metabolism of the bacteria located within this niche is characterized by the breakdown of nitrogen compounds (amino acids, peptides, proteins) derived from gingival crevicular fluid (GCF). This niche possesses a neutral pH, abundance of secondary metabolites, short chain fatty acids (SCFAs), and ammonia., 911,12

In a study, Henne et al. observed that in patients with periodontal disease, Campylobacter rectus could be detected at elevated levels in diseased subgingival sites compared to the healthy control group. ${ }^{55}$ The reverse relationship was observed in the case of Campylobacter concisus. Such microbial shift was accompanied by more frequent isolation of "red complex" bacteria, including $P$. gingivalis $15.9 \%$ vs $0 \%$, F. nucleatum $33.9 \%$, vs $3.1 \%$, and T. forsythia $28.2 \%$ vs $0.1 \%$, respectively. A similar relationship was also observed in another Gram-negative bacterium from the Epsilonproteobacteria class, i.e., H. pylori. Hu et al. investigated the effect of $H$. pylori on inflammation severity and the presence of oral pathogens. ${ }^{26}$ In samples taken from gingival lesions, the incidence of $P$. gingivalis,
P. intermedia, F. nucleatum and T. denticola was significantly higher when $H$. pylori co-existed. This was related to the exacerbation of periodontal disease. Interestingly, the inverse relationship between the presence of $H$. pylori and the pathogenic bacterium $A$. actinomycetemcomitans was also observed. The reason of this phenomenon is not known, but it cannot be ruled out that $H$. pylori can limit the amount of L-lactic acid in the local environment, which is a growth factor for A. actinomycetemcomitans. ${ }^{8}$ The mechanism of competition for lactic acid availability is uncertain and should be confirmed in experimental studies.

\section{Influence of oral $H$. pylori on gastric infections and eradication efficacy}

Colonization of the oral cavity by $H$. pylori can promote reinfections, even when antibiotic therapy has taken place. The ability to form biofilm and coccoid forms by oral $H$. pylori, and the presence of a relatively low biocide concentration in saliva often make it impossible to achieve therapeutic success. In a meta-analysis by Zou et al., it has been shown that in patients with gastric $H$. pylori, the incidence of oral $H$. pylori was significantly higher $(45 \%, 490 / 1088)$ than in those without gastric infection $(23.9 \%, 196 / 821) .{ }^{13}$ It was also determined that the eradication rate of systemic therapy was significantly higher in cases of gastric H. pylori $(85.8 \%, 187 / 218)$ and extremely low for oral $H$. pylori treatment $(5.7 \%, 9 / 158)$. Gao et al. determined the effect of triple therapy (systemic therapy) and triple therapy combined with oral treatment, on the eradication rate of gastric H. pylori. ${ }^{15}$ Combination therapy proved to be more effective, as one year after the end of treatment the incidence of these bacteria was twice as low (32.4\%) as compared to the exclusive use of systemic therapy (62.8\%). A similar relationship, showing an increased degree of $H$. pylori eradication after combination therapy, has been demonstrated in the meta-analyzes of Ren et al. ${ }^{56}$ and Bouziane et al. ${ }^{57}$ It was found that the relative chance of gastric $H$. pylori recurrence in patients who underwent oral and gastric therapy was reduced by $63 \% .^{57}$

\section{Conclusions}

The role of the oral cavity as a reservoir for $H$. pylori is still a controversial issue. There are a great number of articles indicating the presence of this pathogen in the oral cavity, but discrepancies among techniques for $H$. pylori detection and the variations in patients tested often make it difficult to formulate a final verdict. Urease and PCR assays have a high level of positive results in oral $H$. pylori detection, while culture methods have an extremely low sensitivity. Despite large discrepancies in the results 
obtained, it is suggested that the oral cavity may be an extragastric $H$. pylori reservoir, which contributes to reinfections after successful eradication therapies. This location is characterized by the co-existence of numerous microorganisms that naturally colonize the oral cavity. That may potentially affect the physiology and morphology of $H$. pylori. It appears that in the supragingival plaque, $H$. pylori occurs in the form of nonculturable coccoids, which often prevent the detection of such bacteria using standard culture techniques. Subgingival plaque is associated with high amounts of periopathogens, and thus also higher concentrations of AI-2, which determines the presence of $H$. pylori as mixed spiral and coccoid subpopulations. Based on the considerations presented in this review, it is suggested that there is a need to increase the number of studies that identify the relationship between $H$. pylori and other microorganisms, including oral flora. Identification of these mechanisms will make it easier to understand the physiology of oral $H$. pylori and will help to create new, alternative methods to increase the effectiveness of standard systemic therapy.

\section{References}

1. Wu RQ, Zhang DF, Tu E, Chen QM, Chen W. The mucosal immune system in the oral cavity - an orchestra of T cell diversity. Int J Oral Sci. 2014;6:125-132.

2. Linden SK, Sutton P, Karlsson NG, Korolik V, McGuckin MA. Mucins in the mucosal barrier to infection. Mucosal Immunol. 2008;1:183-197.

3. Fábián TK, Hermann P, Beck A, Fejérdy P, Fábián G. Salivary defense proteins: Their network and role in innate and acquired oral immunity. Int J Mol Sci. 2012;13:4295-4320.

4. Frenkel ES, Ribbeck K. Salivary mucins in host defense and disease prevention. J Oral Microbiol. 2015;7.doi: 10.3402/jom.v7.29759.

5. Lindén SK, Wickström C, Lindell G, Gilshenan K, Carlstedt I. Four modes of adhesion are used during Helicobacter pylori binding to human mucins in the oral and gastric niches. Helicobacter. 2008;13:81-93.

6. Flemming $\mathrm{H}-\mathrm{C}$, Wingender J. The biofilm matrix. Nat Rev Microbiol. 2010;8:623-633.

7. Li Y-H, Tian X. Quorum sensing and bacterial social interactions in biofilms. Sensors. 2012;12:2519-2538.

8. Guo L, He X, Shi W. Intercellular communications in multispecies oral microbial communities. Front Microbiol. 2014;5:328.

9. Kolenbrander PE, Palmer RJ, Periasamy S, Jakubovics NS. Oral multispecies biofilm development and the key role of cell-cell distance. Nat Rev Microbiol. 2010;8:471-480.

10. Jenkinson HF. Beyond the oral microbiome. Environ Microbiol. 2011;13:3077-3087.

11. Takahashi N. Microbial ecosystem in the oral cavity: Metabolic diversity in an ecological niche and its relationship with oral diseases. Int Congr Ser. 2005;1284:103-112.

12. Kuboniwa M, Lamont RJ. Subgingival biofilm formation. Periodontol 2000. 2010;52:38-52.

13. Zou QH, Li RQ. Helicobacter pylori in the oral cavity and gastric mucosa: A meta-analysis. J Oral Pathol Med. 2011;40:317-324.

14. Al Sayed A, Anand PS, Kamath KP, Patil S, Preethanath RS, Anil S. Oral cavity as an extragastric reservoir of Helicobacter pylori. ISRN Gastroenterol. 2014;2014:261369.

15. Gao J, Li Y, Wang Q, Qi C, Zhu S. Correlation between distribution of Helicobacter pylori in oral cavity and chronic stomach conditions. J Huazhong Univ Sci Technolog Med Sci. 2011;31:409-412.

16. Khalifa MM, Sharaf RR, Aziz RK. Helicobacter pylori: A poor man's gut pathogen? Gut Pathog. 2010;2:2.

17. Urban J. Helicobacter pylori - characteristics and pathogenic factors. Dent Med Probl. 2010;47:482-486 [in Polish].
18. Biernat M, Gościniak G. The pathogenesis of Helicobacter pylo$r i$ infection - the significance of selected virulence factors. Forum zakaż. 2011;2:7-16 [in Polish].

19. Alm RA, Bina J, Andrews BM, Doig P, Hancock RE, Trust TJ. Comparative genomics of Helicobacter pylori: Analysis of the outer membrane protein families. Infect Immun. 2000;68:4155-4168.

20. Oleastro $M$, Ménard $A$. The role of Helicobacter pylori outer membrane proteins in adherence and pathogenesis. Biology. 2013;2:1110-1134.

21. Fu HW. Helicobacter pylori neutrophil-activating protein: From molecular pathogenesis to clinical applications. World J Gastroenterol. 2014;20:5294-5301.

22. Namavar F, Sparrius M, Veerman EC, Appelmelk BJ, Vandenbroucke-Grauls CM. Neutrophil-activating protein mediates adhesion of Helicobacter pylori to sulfated carbohydrates on highmolecular-weight salivary mucin. Infect Immun. 1998;66:444-447.

23. Hirsch C, Tegtmeyer N, Rohde M, Rowland M, Oyarzabal OA, Backert S. Live Helicobacter pylori in the root canal of endodontic-infected deciduous teeth. J Gastroenterol. 2012;47:936-940.

24. Zhang RG, Duan GC, Fan QT, Chen SY. Role of Helicobacter pylori infection in pathogenesis of gastric carcinoma. World J Gastrointest Pathophysiol. 2016;7:97-107.

25. Aksit Bıcak D, Akyuz S, Kıratlı B, et al. The investigation of Helicobacter pylori in the dental biofilm and saliva samples of children with dyspeptic complaints. BMC Oral Health. 2017;17:67.

26. Hu Z, Zhang Y, Li Z, et al. Effect of Helicobacter pylori infection on chronic periodontitis by the change of microecology and inflammation. Oncotarget. 2016;7:66700-66712.

27. Silva DG, Stevens RH, Macedo JMB, et al. Higher levels of salivary MUC5B and MUC7 in individuals with gastric diseases who harbor Helicobacter pylori. Arch Oral Biol. 2009;54:86-90.

28. Urban J. Helicobacter pylori - diagnostic methods and therapy. Dent Med Probl. 2010;47:487-495 [in Polish].

29. Kalali B, Formichella L, Gerhard M. Diagnosis of Helicobacter pylori: Changes towards the future. Diseases. 2015;3:122-135.

30. Namiot D, Leszczyńska K, Namiot Z, Chilewicz M, Bucki R, Kemona A. The occurrence of Helicobacter pylori antigens in dental plaque; An association with oral health status and oral hygiene practices. Adv Med Sci. 2010;55:167-171.

31. Leszczyńska K, Namiot D, Namiot Z, Leszczyńska J, Jakoniuk $P$, Kemona A. Application of immunoassay for detection of Helicobacter pylori antigens in the dental plaque. Adv Med Sci. 2009;54:194-198.

32. Namiot DB, Leszczyńska $K$, Namiot A, et al. The influence of oral health status and dental plaque removal practices on the occurrence of Helicobacter pylori antigens in saliva. Dent Med Probl. 2013;50:275-281.

33. Urban J, Koszowski R, Płachetka A, Wiczkowski A. An evaluation of selected oral health indicators and cariogenic bacteria titer in patients with Helicobacter pylori. Adv Clin Exp Med. 2017;26:401-407.

34. Kazanowska-Dygdała M, Duś I, Radwan-Oczko M. The presence of Helicobacter pylori in oral cavities of patients with leukoplakia and oral lichen planus. J Appl Oral Sci. 2016;24:18-23.

35. Umeda M, Kobayashi H, Takeuchi Y, et al. High prevalence of Helicobacter pylori detected by PCR in the oral cavities of periodontitis patients. J Periodontol. 2003;74:129-134.

36. Dye BA, Kruszon-Moran D, McQuillan G. The relationship between periodontal disease attributes and Helicobacter pylori infection among adults in the United States. Am J Public Health. 2002;92:1809-1815.

37. Irani S, Monsef Esfahani A, Bidari Zerehpoush F. Detection of Helicobacter pylori in oral lesions. J Dent Res Dent Clin Dent Prospects. 2013;7:230-237.

38. Zijnge V, van Leeuwen MBM, Degener JE, et al. Oral biofilm architecture on natural teet. PLoS One. 2010;5:e9321.

39. Rogers AH, Zilm PS, Diaz PI. Fusobacterium nucleatum supports the growth of Porphyromonas gingivalis in oxygenated and carbondioxide-depleted environments. Microbiol. 2002;148:467-472.

40. Park SA, Ko A, Lee NG. Stimulation of growth of the human gastric pathogen Helicobacter pylori by atmospheric level of oxygen under high carbon dioxide tension. 2011;11:96.

41. Andersen RN, Ganeshkumar N, Kolenbrander PE. Helicobacter pylori adheres selectively to Fusobacterium spp. Oral Microbiol Immunol. 1998;13:51-54. 
42. Ishihara K, Miura T, Kimizuka R, Ebihara Y, Mizuno Y, Okuda K. Oral bacteria inhibit Helicobacter pylori growth. FEMS Microbiol Lett. 1997:152:355-361.

43. Iwatani S, Nagashima H, Reddy R, Shiota S, Graham DY, Yamaoka Y. Identification of the genes that contribute to lactate utilization in Helicobacter pylori. PLoS One. 2014; 9:e103506.

44. Mobley HL, Cortesia MJ, Rosenthal LE, Jones BD. Characterization of urease from Campylobacter pylori. J Clin Microbiol. 1988;26:831-836.

45. Shu M, Browngardt CM, Chen YYM, Burne RA. Role of urease enzymes in stability of a 10-species oral biofilm consortium cultivated in a constant-depth film fermenter. Infect Immun. 2003;71:7188-7192.

46. Okuda K, Ishihara K, Miura T, Katakura A, Noma H, Ebihara Y. Helicobacter pylori may have only a transient presence in the oral cavity and on the surface of oral cancer. Microbiol Immunol. 2000;44:385-388.

47. Khosravi Y, Dieye Y, Loke MF, Goh KL, Vadivelu J. Streptococcus mitis induces conversion of Helicobacter pylori to coccoid cells during coculture in vitro. PLoS One. 2014;9:e112214.

48. Krzyżek P, Gościniak $G$. A proposed role for diffusible signal factors in the biofilm formation and morphological transformation of Helicobacter pylori. Turkish J Gastroenterol. 2018;29:7-13.

49. Wang S, Yang Y, Zhao Y, et al. Sub-MIC tylosin inhibits Streptococcus suis biofilm formation and results in differential protein expression. Front Microbiol. 2016;7:384.

50. Haas B, Grenier D. Isolation, characterization and biological properties of membrane vesicles produced by the swine pathogen Streptococcus suis. PLoS One. 2015;10:e0130528.

51. Linz B, Windsor HM, McGraw JJ, et al. A mutation burst during the acute phase of Helicobacter pylori infection in humans and rhesus macaques. Nat Commun. 2014;5:4165.

52. Krzyżek P. Secretion of outer membrane vesicles as a mechanism promoting H. pylori infections. Post Mikrobiol. 2017;56:316-325.

53. Anderson JK, Huang JY, Wreden C, et al. Chemorepulsion from the quorum signal autoinducer-2 promotes Helicobacter pylori biofilm dispersal. MBio. 2015;6:e00379.

54. Fine DH, Markowitz K, Fairlie K, et al. A consortium of Aggregatibacter actinomycetemcomitans, Streptococcus parasanguinis, and Filifactor alocis is present in sites prior to bone loss in a longitudinal study of localized aggressive periodontitis. J Clin Microbiol. 2013;51:2850-2861.

55. Henne K, Fuchs F, Kruth S, Horz HP, Conrads G. Shifts in Campylobacter species abundance may reflect general microbial community shifts in periodontitis progression. J Oral Microbiol. 2014;6:25874.

56. Ren $Q$, Yan X, Zhou Y, Li WX. Periodontal therapy as adjunctive treatment for gastric Helicobacter pylori infection. In: Yan X, ed. Cochrane Database of Systematic Reviews. Vol 2. Chichester, UK: John Wiley \& Sons, Ltd; 2016:CD009477.

57. Bouziane A, Ahid S, Abouqal R, Ennibi O. Effect of periodontal therapy on prevention of gastric Helicobacter pylori recurrence: A systematic review and meta-analysis. J Clin Periodontol. 2012;39:1166-1173. 